\title{
Classification of the solutions of constant rational semi-dynamical reflection equations
}

\author{
Dedicated to Daniel Arnaudon
}

\author{
Jean Avan ${ }^{1}$ and Geneviève Rollet ${ }^{2}$ \\ Laboratoire de Physique Théorique et Modélisation \\ Université de Cergy-Pontoise, 5 mail Gay-Lussac, Neuville-sur-Oise, \\ F-95031 Cergy-Pontoise Cedex
}

\begin{abstract}
We propose a classification of the solutions $K$ to the semi-dynamical reflection equation with constant rational structure matrices associated to rational scalar RuijsenaarsSchneider model. Four sets of solutions are identified and simple analytic transformations generate all solutions from these sets.
\end{abstract}

\section{Introduction}

Reflection equations appeared in the factorized scattering on a half-line [1, 2]; they describe consistency conditions between reflection matrices $K$ and bulk scattering matrices $R$ guaranteeing integrability of quantum systems with non-periodic boundary conditions. These equations take the form:

$$
R_{12} K_{1} \tilde{R}_{21} K_{2}=K_{2} \tilde{R}_{12} K_{1} R_{21} \text {. }
$$

As usual, the auxiliary spaces indexed by $(1,2)$ may be loop spaces $V \otimes \mathbb{C}(\lambda)$ and the spectral parameter dependence of $R$ and $K$ is then implicit.

General quadratic exchange algebras were soon considered 3, 4] stemming in particular from the study of quantum integrable non-ultra-local field theories [5]. Their general form reads:

$$
A_{12} K_{1} B_{12} K_{2}=K_{2} C_{12} K_{1} D_{12}
$$

with consistency conditions: unitarity of $A$ and $D, C_{12}=B_{21}$, Yang-Baxter type cubic equations for $A, B, C, D$.

They have been the object of many studies, running from their covariance properties [3], their interpretation as twists of quantum groups (non-affine) [6] to the classification of solutions $K$ for a variety of $A, B, C, D$ matrices (see [3, 7, 8, 9, 10]) to the use of explicit $K$-solutions to

\footnotetext{
${ }^{1}$ e-mail: avan@u-cergy.fr

${ }^{2}$ e-mail: rollet@u-cergy.fr
} 
build integrable spin chains with integrable boundary (see [2]) and the study of their symmetry properties [11].

Similarly to the Yang-Baxter equation, the generalized reflection equations admit "dynamical" extensions. Precisely, the structure matrices $A, B, C, D$ and the reflection matrix $K$ then depend on parameters $\left\{\lambda_{i}\right\}$ interpreted as coordinates on the dual of a Lie Algebra $\mathfrak{h}$ (often abelian, although the notion of non-abelian quantum dynamical group exists, see e.g. [12, 13, 14]). The consistency equations for $A, B, C, D$ and the quadratic equation for $K$ now occur with consistent shifts on these parameters, and zero-weight compatibility conditions under adjoint action of $\mathfrak{h}$ on $A, B, C, D$. Contrary to the quantum-group case, where only one extension is identified (Gervais-Neveu-Felder equation, see [15, 16]), at this time two unequivalent dynamical extensions of (1.2) have been found.

The first one, known as either "boundary dynamical quantum group" [17] or "fully dynamical", can be deduced from (1.2) by the famous vertex-IRF transformation [18]. It has been studied quite extensively, with construction of solutions [17], interpretation as twist [19], construction of quantum integrable systems à la Gaudin [20]; recently the general form of quantum traces, and local spin chains associated to it, was described [21]. The equation reads:

$$
A_{12} K_{1}\left(\lambda+h_{2}\right) B_{12} K_{2}\left(\lambda+h_{1}\right)=K_{2}\left(\lambda+h_{1}\right) C_{12} K_{1}\left(\lambda+h_{2}\right) D_{12},
$$

with zero weight conditions for the four structure matrices $\left(A_{12}, B_{12}, C_{12}\right.$ and $D_{12}$ commute with $\left.h_{1}+h_{2}\right)$.

The second dynamical extension of (1.2), which we will consider here, has been characterized only recently [22]. Originally found in the quantization of the scalar RuijsenaarsSchneider $r$-matrix structure [23], it received its general formulation and analysis of comodule and 'coproduct-like' properties in [22]; quantum traces and local spin-chain Hamiltonians were discussed in [21]. The quadratic exchange relation has the form:

$$
A_{12} K_{1} B_{12} K_{2}\left(\lambda+h_{1}\right)=K_{2} C_{12} K_{1}\left(\lambda+h_{2}\right) D_{12}
$$

with $C_{12}=B_{21}$ and zero weight conditions for only two structure matrices (precisely $B_{12}$ commutes with $h_{1}$ and $D_{12}$ with $h_{1}+h_{2}$ ).

Very few examples are known, in fact only the quantization of scalar Ruijsenaars-Schneider model provides at this time explicit forms of $A, B, C, D$ and $K$ matrices. It is however clear that in order to both understand the algebraic meaning of that structure, and to use it in explicit constructions of integrable Hamiltonians [21] we must find a general classification of solutions.

More precisely, the problem is twofold: find $A, B, C, D$ constrained by a set of Y.B.-like equations compatible with the associativity of the algebra; find $K$ for such $A, B, C, D$ 's. Contrarily to the Y.B. case, the two questions are decoupled. Note that several distinct forms of YB-type equations may arise as sufficient conditions, noticeably when a spectral parameter dependance occurs [23]. This will be commented upon in the conclusion.

We shall here restrict ourselves to the "simplest" second problem: for a given set of rational, non-spectral parameter dependent (called here "constant" in the usual $R$-matrix terminology) $A, B, C, D$, find all possible reflection matrices $K$. The set of $A, B, C, D$ we will start with is the one obtained in [23] describing the Lax matrix structure of quantum rational RuijsenaarsSchneider model [24].

First of all we shall describe two general properties of equation (1.4), including two possible ways of constructing extra solutions from a given one: a gauge transformation property and a decoupling "column degeneracy" property (actually a limit of the first one). 
We shall then identify four sets of meromorphic solutions $K$ exhausting the set of solutions. The solution in 23] is of course recovered, but it is understood in a more general formulation, and in addition three other types of solutions are exhibited, one invertible and two rank one projectors.

We shall finally discuss the interpretation of our new solutions, possible extensions to trigonometric, elliptic cases, constant and non-constant, and come back to the question of finding new $A, B, C, D$ 's.

\section{General properties of constant semi-dynamical reflec- tion equations}

Let us recall that the semidynamical quadratic exchange relation without spectral parameter reads:

$$
\begin{aligned}
& A_{12} K_{1} B_{12} K_{2}\left(\lambda+h_{1}\right)=K_{2} B_{21} K_{1}\left(\lambda+h_{2}\right) D_{12} \\
& \text { with }\left[B_{12}, h_{1}\right]=0 \quad \text { and } \quad\left[D_{12}, h_{1}+h_{2}\right]=0 .
\end{aligned}
$$

We shall assume that the representation space $V$ of the matrices $K$ (considering a representation space $V \otimes V$ for $A, B, C, D$ matrices) is a diagonalizable irreducible module of $\mathfrak{h}$. As a consequence we shall identify $\mathfrak{h}$ with the Cartan algebra of $g l(n)$ and $V$ with $\mathbb{C}^{n}$. The set of equations (2.1), projected on generators $e_{i j} \otimes e_{k l}$ of $g l(n)$, then takes the form:

$$
\sum_{x y} M_{x y}^{i j k l} K_{x j} K_{y l}\left(\lambda_{j}+\gamma\right)=K_{k l} \sum_{x} N_{x}^{i j l} K_{x j}\left(\lambda_{l}+\gamma\right)
$$

with $M$ and $N$ quadratically depending on $A, B, C, D$ matrix elements. The notation $f\left(\lambda_{j}+\gamma\right)$ will denote the adjoint action of the shift operator $e^{\gamma \partial_{j} 3}$ on any function $f$ of the variables $\lambda_{1}, \ldots, \lambda_{n}$.

Two general properties of (2.1) now follow:

\section{Property 1}

The set of equations (2.1) subdivides into subsets coupling only two fixed columns of $K$ (namely those with indices $j$ and $l$ in (2.2) ).

\section{Property 2}

For a given set of $A, B, C, D$ matrices, if $K$ is a solution of (2.1) then for any function $f$ of the variables $\lambda_{1}, \ldots, \lambda_{n}$, another solution is given by $\tilde{K}$, with

$$
\tilde{K}_{i j}=K_{i j} \frac{f\left(\lambda_{j}+\gamma\right)}{f(\lambda)}
$$

In other words, multiplying a solution $K$ on the right by a diagonal matrix, $\left(f_{j}(\lambda)\right)$, satisfying the flatness condition

$$
f_{j}(\lambda) f_{i}\left(\lambda_{j}+\gamma\right)=f_{i}(\lambda) f_{j}\left(\lambda_{i}+\gamma\right)
$$

$$
{ }^{3} \partial_{j}=\frac{\partial}{\partial \lambda_{j}}
$$


is a gauge transformation; the solution of the flatness equations (2.4) being precisely given by a gradient:

$$
f_{j}(\lambda)=\frac{f\left(\lambda_{j}+\gamma\right)}{f(\lambda)}
$$

Indeed, the cases where some $f_{j}$ are equal to zero -perfectly admissible solutions of (2.4)- can actually be included in this gradient formulation: $f=\prod_{j} e^{c_{j} \lambda_{j} / \gamma}$ yields $f_{j}(\lambda)=\frac{f\left(\lambda_{j}+\gamma\right)}{f(\lambda)}=e^{c_{j}}$ and finally $\lim _{c_{j} \rightarrow-\infty} f_{j}(\lambda)=0$. As a consequence, one can multiply any solution $K$ on the right by a constant diagonal matrix, allowing for instance to set to zero any number of chosen columns.

In the case when the matrix $D$ is not only zero-weight but also "dynamical zero-weight" $\left(\left[h_{1} \partial_{1}+h_{2} \partial_{2}, D_{12}\right]=0\right)$, equation (1.4) can be rewritten without shifts, albeit with non-abelian $K$-matrices: $\tilde{K}_{1}=K_{1} e^{\gamma \partial_{1}}$. Gauge transformation (2.3) can then be interpreted as a canonical transformation redefining $e^{\gamma \partial_{j}}$ as $f^{-1}(\lambda) e^{\gamma \partial_{j}} f(\lambda)$, that is, $\frac{f\left(\lambda_{j}+\gamma\right)}{f(\lambda)} e^{\gamma \partial_{j}}$.

\section{Solving the rational case}

In the rational constant case derived in [23] the $A, B, C, D$ matrices read:

$$
\begin{array}{r}
A=\mathbb{1}+\sum_{i \neq j} \frac{\gamma}{\lambda_{i j}}\left(e_{i i}-e_{i j}\right) \otimes\left(e_{j j}-e_{j i}\right) \\
B=\mathbb{1}+\sum_{i \neq j} \frac{\gamma}{\lambda_{i j}-\gamma} e_{j j} \otimes\left(e_{i i}-e_{i j}\right)=C^{\pi} \\
D=\mathbb{1}+\sum_{i \neq j} \frac{\gamma}{\lambda_{i j}}\left(e_{i j} \otimes e_{j i}-e_{i i} \otimes e_{j j}\right)
\end{array}
$$

Equations (2.2) take different forms, depending on the specific choice of generator $e_{i j} \otimes e_{k l}$. A priori 15 different cases would have to be considered $(i=j=k=l, i=j=k \neq l, \ldots,(i, j, k, l)$ all distinct); in fact, some equations directly reduce to $0=0$, some equations actually coincide and some are consequences of a set of others. The case $i=j=k=l$ collapses to $0=0$; the set of 7 cases involving exactly two distinct indices reduces to the following three functional equations:

$$
\begin{array}{r}
\frac{\gamma}{\lambda_{i j}} K_{i j} K_{i j}\left(\lambda_{j}+\gamma\right)=K_{i j} K_{j j}\left(\lambda_{j}+\gamma\right)-\left(1-\frac{\gamma}{\lambda_{i j}}\right) K_{j j} K_{i j}\left(\lambda_{j}+\gamma\right) \\
\frac{\gamma}{\lambda_{i j}} K_{i j} K_{j i}\left(\lambda_{j}+\gamma\right)=K_{i j} K_{i i}\left(\lambda_{j}+\gamma\right)-\left(1+\frac{\gamma}{\lambda_{i j}}\right) K_{i i} K_{i j}\left(\lambda_{i}+\gamma\right) \\
\left(2-\frac{\gamma}{\lambda_{i j}}\right) K_{i j} K_{j i}\left(\lambda_{j}+\gamma\right)=K_{i j} K_{i i}\left(\lambda_{j}+\gamma\right)-\left(1-\frac{\gamma}{\lambda_{i j}}\right) K_{i i} K_{i j}\left(\lambda_{i}+\gamma\right)+ \\
\left(1-\frac{\lambda_{i j}}{\gamma}\right)\left(K_{i i} K_{j j}\left(\lambda_{i}+\gamma\right)-K_{j j} K_{i i}\left(\lambda_{j}+\gamma\right)\right)
\end{array}
$$

Actually the set of equations (3.2) decouples since it's the only one among the three sets of equations involving a shift on the variable labeled by the column index. It describes self-coupling of a single column. Let us consider this set of equations first. One immediatly notices that, if the diagonal term $K_{j_{0} j_{0}}$ vanishes (as a function), then any other term of the same column also vanishes $^{4}\left(\forall i, K_{i j_{0}}=0\right)$. Inserting this into (2.2) yields two types of equations:

\footnotetext{
${ }^{4}$ We reduce our analysis to meromorphic functions.
} 
-either one with $j$ or $l$ equal to $j_{0}$, that reduces to $0=0$.

-or one with none of the column indices equal to $j_{0}$, in this case no term $K_{i j_{0}}$ is involved in the equation.

This leads us to introduce the set $J_{0}$ of all indices $j_{0}$ such that $K_{j_{0} j_{0}}=0$. Therefore the set of equations (2.2) reduces to the subset with no zero-column. We shall now solve this subset of equations, and from now on it will be understood that neither $j$ nor $l$ belong to $J_{0}$. Introducing the following natural gauge-invariants:

$$
X_{i j}=\frac{K_{i j}}{K_{j j}} ; \quad R_{j l}=\frac{K_{j j}\left(\lambda_{l}+\gamma\right)}{K_{j j}} \frac{K_{l l}}{K_{l l}\left(\lambda_{j}+\gamma\right)},
$$

we get:

$$
\begin{gathered}
X_{i j}\left\{\alpha_{k j} \alpha_{i k} X_{k l}(j)+\left(\alpha_{i k}\left(\alpha_{i j}-\alpha_{k j}\right)-\alpha_{i j}+1\right) X_{j l}(j)-\alpha_{i j}\left(\alpha_{i k}-1\right) X_{i l}(j)\right\} \\
-X_{k j}\left\{\alpha_{k j}\left(\alpha_{i k}-1\right) X_{k l}(j)+\left(\alpha_{i k}\left(\alpha_{i j}-\alpha_{k j}\right)+\alpha_{k j}\right.\right. \\
\left.\left.+\alpha_{l j}\left(1-\alpha_{i j}\right)-1\right) X_{j l}(j)+\alpha_{i j}\left(\alpha_{l j}-\alpha_{i k}\right) X_{i l}(j)\right\} \\
=\alpha_{l j} R_{j l} X_{k l}\left\{\alpha_{i l} X_{i j}(l)-\left(\alpha_{i l}-1\right) X_{l j}(l)\right\}
\end{gathered}
$$

introducing here a short-hand notation $(j)$ for $\left(\lambda_{j}+\gamma\right)$ and:

$$
\alpha_{a b}=1+\left(1-\delta_{a b}\right) \frac{\gamma}{\lambda_{a b}\left(\lambda_{j}+\gamma, \lambda_{l}+\gamma\right)}
$$

Before actually solving equations (3.6), let us first describe the reconstruction procedure of $K_{i j}$ from $R_{j l}$ and $X_{i j}$. It is done in two steps:

-1- Exhibiting some diagonal part $K_{j j}$ leading to the found $R_{j l}$. This requires that $R_{j l}$ obey a zero-curvature condition:

$$
\frac{R_{i j}\left(\lambda_{k}+\gamma\right)}{R_{i j}} \frac{R_{j k}\left(\lambda_{i}+\gamma\right)}{R_{j k}} \frac{R_{k i}\left(\lambda_{j}+\gamma\right)}{R_{k i}}=1
$$

which may not be satisfied for some particular solution $R_{j l}$ of (3.6). In such a case, no associated $K$ exists. If, on the other hand, some $K_{j j}(\lambda)$ yield $R_{j l}$, all other solutions for this $R_{j l}$ are given by $\tilde{K}_{j j}(\lambda)=f_{j}(\lambda) K_{j j}(\lambda),\left(f_{j}(\lambda)\right)$ being a flat (i.e. satisfying the flatness equations (2.4), hence $\left.f_{j}(\lambda)=f\left(\lambda_{j}+\gamma\right) / f(\lambda)\right)$ diagonal matrix

-2- Finally $K_{i j}(\lambda)=X_{i j}(\lambda) K_{j j}(\lambda)$.

Let us now come back to the resolution of equations (3.6). We will once again first concentrate on equation (3.2) which involves only $X$ :

$$
\frac{\gamma}{\lambda_{i j}} X_{i j} X_{i j}\left(\lambda_{j}+\gamma\right)=X_{i j}-\left(1-\frac{\gamma}{\lambda_{i j}}\right) X_{i j}\left(\lambda_{j}+\gamma\right)
$$

It takes the simple form $G_{i j}\left(\lambda_{j}+\gamma\right)=G_{i j}$ introducing the invertible parametrization $X_{i j}=$ $1+\frac{\lambda_{i j}}{G_{i j}-\lambda_{i j}}$.

The next equation we will take into account is equation (3.6) with $j=l^{5}$. It involves only entries of $G$ in a single column $j$ and with shifts only on $\lambda_{j}$, precisely the variable for which $G$ is $\gamma$-periodic. This equation with three distinct indices actually factorizes in the remarkably nice following way:

$$
\left(G_{i j}-G_{k j}\right)\left(G_{i j}-G_{k j}-\lambda_{i k}\right)=0 .
$$

\footnotetext{
${ }^{5}$ This requires that $n \geq 3$, we will come back to $n=2$ afterward.
} 
The solutions of this last equation read:

$$
G_{i j}=G_{j}+\frac{1}{2}\left(1+\epsilon_{j}\right) \lambda_{i}
$$

where $\epsilon_{j}$ is a sign and $G_{j}$ an arbitrary function. The periodicity condition that $G_{i j}$ has to satisfy is transmitted to $G_{j}: G_{j}\left(\lambda_{j}+\gamma\right)=G_{j}$.

To get suitable consistency relations between these $G_{j}$ 's, we now have to consider equations coupling two different non zero columns. The simplest such equations are (3.3) and (3.4). Manipulating them, for column indices $j$ and $l$ not in $J_{0}$, yields:

$$
\begin{aligned}
\left(R_{j l}-\rho_{j l}\right)\left(G_{j l}-G_{l j}\right) & =0 \\
\left(R_{j l}-\rho_{j l}\right)\left(\left(G_{j l}-\lambda_{j l}\right)\left(G_{l j}+\lambda_{j l}\right)-\gamma / 2\left(G_{j l}+G_{l j}\right)\right) & =\gamma\left(R_{j l}+\rho_{j l}\right)\left(G_{l j}-G_{j l}+\lambda_{j l}\right)
\end{aligned}
$$

where $\rho_{j l}=\frac{\lambda_{j l}+\gamma}{\lambda_{j l}-\gamma}$.

At this point the set of solutions splits into two cases: -case I

$$
G_{j l}=G_{l j}+\lambda_{j l} \quad \text { and } \quad R_{j l}=\rho_{j l}, \quad \text { that is } \quad K_{j j}=\frac{f\left(\lambda_{j}+\gamma\right)}{f} \prod_{l \neq j} \frac{\gamma}{\lambda_{j}-\lambda_{l}}
$$

-case II

$$
G_{j l}=G_{l j} \quad \text { and } \quad R_{j l}=\rho_{j l} \frac{\left(G_{j l}-\lambda_{j l}\right)\left(G_{j l}+\lambda_{j l}-\gamma\right)}{\left(G_{j l}+\lambda_{j l}\right)\left(G_{j l}-\lambda_{j l}-\gamma\right)}
$$

Let us note that at this stage we cannot guarantee for case II that such an $R_{j l}$ actually correspond to the diagonal part of some $K$ matrix (the definition of $R$ in equation (3.5) is not necessarily invertible; as already mentioned this invertibility requires an extra zero-curvature condition on $R$ ).

Plugging back the form (3.8) of $G_{i j}$ into equations (3.10) and (3.11) gives $G_{j}=G+\frac{1}{2}(\sigma+$ $\left.\sigma^{\prime}+\epsilon_{j_{1}}-\epsilon_{j}\right) \lambda_{j}$ with $G$ an arbitrary function, $j_{1}$ the label of some non-zero column, $\sigma$ and $\sigma^{\prime}$ two signs obeying the following constraints: $\forall j \neq l,\left(\sigma+\sigma^{\prime}+\epsilon_{j_{1}}-\epsilon_{j}-\epsilon_{l}\right)^{2}=1$.

Note that setting $j=j_{1}$ in this constraint reads $\forall l \neq j_{1},\left(\sigma+\sigma^{\prime}-\epsilon_{l}\right)^{2}=1$; the constraints can thus be factorized: $\left(\epsilon_{j_{1}}-\epsilon_{j}\right)\left(\epsilon_{j_{1}}-\epsilon_{j}+2\left(\sigma+\sigma^{\prime}-\epsilon_{l}\right)\right)=0$.

Here again the set of solutions splits:

-either $\forall j, \epsilon_{j}=\epsilon_{j_{1}}$ (homogeneous case) and $G_{j}=G+\frac{1}{2}\left(\sigma+\sigma^{\prime}\right) \lambda_{j}$ with constraints $\left(\sigma+\sigma^{\prime}-\right.$ $\left.\epsilon_{j_{1}}\right)^{2}=1$, that is $\left(\sigma+\sigma^{\prime}\right)\left(\sigma+\sigma^{\prime}-2 \epsilon_{j_{1}}\right)=0$ or equivalently $\sigma+\sigma^{\prime}=\left(1+\sigma^{\prime \prime}\right) \epsilon_{j_{1}}$ with $\sigma^{\prime \prime}$ a sign, leading finally to:

$$
\begin{array}{r}
G_{i j}=G+\frac{1}{2}\left(\epsilon+\epsilon^{\prime}\right) \lambda_{j}+\frac{1}{2}(1+\epsilon) \lambda_{i}, \text { with } G\left(\lambda_{j}+\gamma\right)=G-\frac{1}{2}\left(\epsilon+\epsilon^{\prime}\right) \gamma \\
\epsilon \text { and } \epsilon^{\prime} \text { being two arbitrary signs }\left(\epsilon=\epsilon_{j_{1}} \text { and } \epsilon^{\prime}=\sigma^{\prime \prime} \epsilon_{j_{1}}\right) .
\end{array}
$$

-or there exists some $j_{2}$ such that $\epsilon_{j_{2}} \neq \epsilon_{j_{1}}$ (inhomogeneous case) then $\forall j \notin\left\{j_{1}, j_{2}\right\}, \epsilon_{j_{1}}+$ $\left(\sigma+\sigma^{\prime}-\epsilon_{j}\right)=0$. Again we have to consider two options:

*either there exists some $j_{3} \notin\left\{j_{1}, j_{2}\right\}$ such that $\epsilon_{j_{3}}=\epsilon_{j_{1}}$, then $\sigma+\sigma^{\prime}=0$ and $\forall j \neq j_{2}, \epsilon_{j}=$ $\epsilon_{j_{1}}$.

${ }^{*}$ or $\forall j \neq j_{1}, \epsilon_{j}=-\epsilon_{j_{1}}$ and $\sigma+\sigma^{\prime}=-2 \epsilon_{j_{1}}$. 
In fact, these two cases merge into one, characterized by one special column labelled by $j_{0}$, such that $\forall j \neq j_{0}, \epsilon_{j}=-\epsilon_{j_{0}}$, then $\forall j, G_{j}=G-\frac{1}{2}\left(\epsilon_{j_{0}}+\epsilon_{j}\right) \lambda_{j}$ and:

$$
G_{i j}=G-\frac{1}{2}\left(\epsilon_{j_{0}}+\epsilon_{j}\right) \lambda_{j}+\frac{1}{2}\left(1+\epsilon_{j}\right) \lambda_{i}=\left\{\begin{array}{l}
\forall j \neq j_{0}, G_{i j}=G+\frac{1}{2}\left(1-\epsilon_{j_{0}}\right) \lambda_{i} \\
G_{i j_{0}}=G-\epsilon_{j_{0}} \lambda_{j_{0}}+\frac{1}{2}\left(1+\epsilon_{j_{0}}\right) \lambda_{i}
\end{array}\right.
$$

with $\forall j \neq j_{0}, G\left(\lambda_{j}+\gamma\right)=G$ and $G\left(\lambda_{j_{0}}+\gamma\right)=G+\epsilon_{j_{0}} \gamma$.

However, reinserting the two forms (3.12) and (3.13) in the equation with three distinct indices obtained from (3.6) letting $i=j=j_{0}$ rules out the inhomogeneous form (3.13) and validates (3.12).

From (3.12) one has: $G_{j l}-G_{l j}=\frac{1}{2}\left(1-\epsilon^{\prime}\right) \lambda_{j l}$, so case I (3.10) corresponds to $\epsilon^{\prime}=-1$ and case II (3.11) to $\epsilon^{\prime}=1$. Now one can propose a complete expression both for $G$ and the diagonal part of $\mathrm{K}$ (already given in (3.10) for case I). Given $f$ and $g$ as any two meromorphic functions such that $\forall j, g\left(\lambda_{j}+\gamma\right)=g$, one gets:

-case Ia $\left(\epsilon=1, \epsilon^{\prime}=-1, g=G\right)$

$$
G_{i j}=g+\lambda_{i}, \quad \text { and } K_{j j}=\frac{f\left(\lambda_{j}+\gamma\right)}{f} \prod_{l \neq j} \frac{\gamma}{\lambda_{j}-\lambda_{l}}
$$

-case Ib $\left(\epsilon=-1, \epsilon^{\prime}=-1, g=G-\sum_{j} \lambda_{j}\right)$

$$
G_{i j}=g+\Sigma_{j} \text { with } \Sigma_{j}=\sum_{l \neq j} \lambda_{l} \text { and } K_{j j}=\frac{f\left(\lambda_{j}+\gamma\right)}{f} \prod_{l \neq j} \frac{\gamma}{\lambda_{j}-\lambda_{l}}
$$

-case IIa $\left(\epsilon=1, \epsilon^{\prime}=1, g=G+\sum_{j} \lambda_{j}\right)$

$$
\begin{gathered}
G_{i j}=g-\Sigma_{j}+\lambda_{i} \text { with } \Sigma_{j}=\sum_{l \neq j} \lambda_{l} \quad \text { and } R_{j l}=\frac{\lambda_{j l}+\gamma}{\lambda_{j l}-\gamma} \frac{\left(g-\Sigma_{l}+\lambda_{l}\right)\left(g-\Sigma_{j}+\lambda_{j}-\gamma\right)}{\left(g-\Sigma_{j}+\lambda_{j}\right)\left(g-\Sigma_{l}+\lambda_{l}-\gamma\right)} \\
\text { that is } K_{j j}=\frac{f\left(\lambda_{j}+\gamma\right)}{f}\left(g-\Sigma_{j}+\lambda_{j}\right) \prod_{l \neq j} \frac{\gamma}{\lambda_{j}-\lambda_{l}}
\end{gathered}
$$

-case $\operatorname{IIb}\left(\epsilon=-1, \epsilon^{\prime}=1, g=G\right)$

$$
\begin{aligned}
& G_{i j}=g \quad \text { and } \quad R_{j l}=\frac{\lambda_{j l}+\gamma}{\lambda_{j l}-\gamma} \frac{\left(g-\lambda_{j l}\right)\left(g+\lambda_{j l}-\gamma\right)}{\left(g+\lambda_{j l}\right)\left(g-\lambda_{j l}-\gamma\right)} \\
& \text { that is } K_{j j}=\frac{f\left(\lambda_{j}+\gamma\right)}{f} \prod_{l \neq j} \frac{g+\lambda_{j}-\lambda_{l}}{\lambda_{j}-\lambda_{l}}
\end{aligned}
$$

Finally, we check that these four sets (3.14, 3.15, 3.16, 3.17) actually verify all the required equations.

Let us now come back to the question of the zero columns. We recall that starting from the introduction of $X$ and $R$ in (3.5), we have restricted our analysis to the non-zero columns of $K\left(j, l \notin J_{0}\right)$; in particular any sum or product in (3.14, 3.15, 3.16, 3.17) has to be understood within this set of non-zero columns; the same holds for the periodicity conditions on $g$. We are 
actually able to reincorporate the indices of $J_{0}$ into the products appearing in the expressions of $K_{j j}$ using the following gauges:

Cases Ia, Ib and IIa $\quad f=\prod_{j_{0} \in J_{0}, j \notin J_{0}} \frac{1}{\Gamma\left(\frac{\lambda_{j}-\lambda_{j_{0}}}{\gamma}\right)}$ yields $\frac{f\left(\lambda_{j}+\gamma\right)}{f}=\prod_{j_{0} \in J_{0}} \frac{\gamma}{\lambda_{j}-\lambda_{j_{0}}}$

Case IIb $f=\prod_{j_{0} \in J_{0}, j \notin J_{0}} \frac{\Gamma\left(\frac{g+\lambda_{j}-\lambda_{j_{0}}}{\gamma}\right)}{\Gamma\left(\frac{\lambda_{j}-\lambda_{j_{0}}}{\gamma}\right)}$ yields $\frac{f\left(\lambda_{j}+\gamma\right)}{f}=\prod_{j_{0} \in J_{0}} \frac{g+\lambda_{j}-\lambda_{j_{0}}}{\lambda_{j}-\lambda_{j_{0}}}$.

These $J_{0}$-indices can then be reintroduced in $\Sigma_{j}$ (cases Ib and IIa), adding $\sum_{l \in J_{0}} \lambda_{l}$ to $\Sigma_{j}$, up to a redefinition of $g$, which is compatible with the periodicity conditions of $g$ since they are only required on the variables $\left\{\lambda_{i}\right\}, i \notin J_{0}$. From these manipulations, one can see that any solution with zero-columns indexed by $J_{0}$ can be obtained from a solution with no zero-column, first by applying a gauge transformation (multiplication on the right by the diagonal matrix with 0 for indices in $J_{0}$ and 1 for other indices) and then dropping the periodicity conditions for $g$ on the variables $\left\{\lambda_{j_{0}}, \forall j_{0} \in J_{0}\right\}$.

Another restriction has been introduced in the course of this section to get the solutions (3.14, 3.15, 3.16, 3.17). The use of equation (3.7) lead us to suppose $n \geq 3$. In fact one can directly check that these solutions still are the full set of solutions for $n=2$, the only noticeable point being that for $n=2, \Sigma_{j}=\lambda_{i}($ for $i \neq j$ ) and consequently cases (a) and (b) collapse both for cases I and II.

We can now fully classify the solutions of the constant rational semi-dynamical reflection equations.

\section{Solutions of the constant rational semi-dynamical re- flection equations}

The solutions of the semi-dynamical reflection equations (2.1) with rational constant structure matrices (3.1) belong to four sets, defined by the following formulae up to two procedures:

- a gauge transformation (2.3)

- or a multiplication on the right by the diagonal gauge matrix $\left(d_{j j}^{J_{0}}\right)\left(\forall j \in J_{0}, d_{j j}^{J_{0}}=0\right.$ and $\left.\forall j \notin J_{0}, d_{j j}^{J_{0}}=1\right)$ and the elimination of the periodicity conditions on the corresponding variables $\left\{\lambda_{j_{0}}\right\}_{j_{0} \in J_{0}}$ for $g$.

In the following formulae $g$ denotes a meromorphic function $\gamma$-periodic on each $\lambda_{k}$.

Case Ia:

$$
K_{i j}=\frac{g+\lambda_{i}}{g+\lambda_{j}} \prod_{k \neq j} \frac{\gamma}{\lambda_{j}-\lambda_{k}}
$$

Case Ib:

$$
K_{i j}=\frac{g+\Sigma_{j}}{g+\Sigma_{i}} \prod_{k \neq j} \frac{\gamma}{\lambda_{j}-\lambda_{k}}
$$

Case IIa:

$$
K_{i j}=\left(g-\Sigma_{i j}\right) \prod_{k \neq j} \frac{\gamma}{\lambda_{j}-\lambda_{k}} \quad \text { with } \quad \Sigma_{i j}=\sum_{k} \lambda_{k}-\lambda_{i}-\lambda_{j}
$$

Case IIb:

$$
K_{i j}=\frac{g}{g-\lambda_{i}+\lambda_{j}} \prod_{k \neq j} \frac{g+\lambda_{j}-\lambda_{k}}{\lambda_{j}-\lambda_{k}}
$$

The last solution (case IIb) is equivalent to the one in [23], once the fully periodic $g$ function is turned into a constant (from the view point of (2.1) there is no difference between a constant 
and a function $\gamma$-periodic with respect to each variable). It thus generates, by the quantum trace construction given in [26, commuting Hamiltonians of scalar $A_{n}$ Ruijsenaar-Schneider model.

Solutions of cases Ia and Ib are rank-one matrices, use of which to build commuting Hamiltonians is not clear (as far as the first Hamiltonian is concerned, we recover the $g \rightarrow \infty$ limit of the (case IIb) scalar $A_{n}$ Ruijsenaar-Schneider model). They may have to be understood within a larger structure of type "reflection-transmission" [27] (still to be formulated).

Finally, solution IIa yields, by the same quantum trace construction, commuting $N$-body Hamiltonians, the first one being:

$$
H=\sum_{j \in\{1 \cdots N\}} e^{p_{j}}\left(g-\sum_{k} \lambda_{k}+2 \lambda_{j}\right) \prod_{k \neq j} \frac{\gamma}{\lambda_{j}-\lambda_{k}} .
$$

\section{Conclusion and prospective}

Now that a full classification is achieved for the simplest constant rational semi-dynamical reflection equation, it is not to be expected that the trigonometric case yield qualitatively different classifications of solutions. The elliptic case, if any, is unclear: indeed dynamical constant elliptic $R$ matrices are essentially unknown except for the algebras $A_{1}$ and $A_{2}$ [28] and are known to be of a more complicated (and not explicitely known) form for generic Lie algebras $A_{n}$.

The next questions to address in the study of this algebraic structure will be the following:

\subsection{Extension to existent non-constant $A B C D$ matrices}

Known non constant rational and trigonometric $A B C D$ matrices exhibit a simple decoupling between the constant part and the simple-pole contributions; a fact which should be very helpful in disentangling the corresponding reflection equations. Elliptic non-constant solutions by contrast exhibit a coupling between spectral and dynamical parameters, but the explicit knowledge of trigonometric solutions may help to guess the form of some elliptic solutions. It is to be emphasized here that the Yang-Baxter equations for $A B C[23$ exhibit shifts in the spectral parameters; in fact the actual $R$ matrices constructed by Arutyunov et al. must be defined as containing explicit differential operators, e.g $A_{12} \equiv e^{\frac{d}{d z_{2}}} a_{12} e^{\frac{-d}{d z_{1}}}$ where $a_{12}$ is the c-number matrix appearing in the reflection equation. These shifts are related to a more general gauge covariance of the YB and reflection equations, by l.h.s. multiplication by a diagonal operator involving differential operators in the spectral parameters (used together with the gauge invariance by r.h.s. multiplication used here (2.3) $)$. Occurence of these differential operators is not surprising here, since the derivative w.r.t. the spectral parameter is an evaluation representation of the $d$ operator which completes the Cartan algebra of diagonal matrices when considering the affine Lie algebra $\hat{A_{n}}$ instead of the finite Lie algebra $A_{n}$. Note that the zero-weight conditions obeyed by the $A B C D$ matrices also involve (on the explicit example of Arutyunov et al.) zeroweight conditions for $D, B, C$ under the $d$ operator. (e.g. $D \equiv D\left(z_{1}-z_{2}\right)$ ). Interestingly the YB equation for $D$ never acquires shifts on the spectral parameters under these gauge transformations due to this particular $d$-zero weight condition.

Incidentally this leads us to note that in the affine case one may add to the already introduced dynamical variables $\lambda_{i}$ an extra dynamical coordinate associated to $d$. The semi- 
dynamical reflection equation may thus acquire an extra "dynamics" (yet to be defined) similar somehow to the shift on the elliptic module in the affine elliptic algebras of Jimbo et al. 29.

\subsection{Construction of new $A B C D$ sets}

The problem is to get new solutions of the YB consistency equations with the canonical zeroweight conditions (including w.r.t. $d$ if necessary). A possible programme for constructing (and possibly classifying) new sets of such $A B C D$ coefficient matrices for semi-dynamical reflection equations starts from the already known classification of Gervais-Neveu-Felder solutions (matrices $D$ ). Indeed they are the best controlled algebraic objects, obeying the GNF equation, with canonical zero-weight conditions and dependance on the difference of the spectral parameters. In addition, contrary to the matrices $A, B, C$ their YB equation is unambiguously gauge-invariant w.r.t. the left gauge multiplication, as discussed above. From a given $D$ one should then deduce classes of $B$ and $C$ matrices, and finally $A$ matrices. It is not clear whether the unit-solution condition $A B=C D$ must be imposed a priori. It is not necessarily consistent with the YB equations; however it is crucial in guaranteeing that the spin chains built from such semi-dynamical reflection representations have a local interaction term up to the "dynamical" modification, see [21]. Its exact interpretation remains to be clarified, and the explicit construction of new classes of solution may also cast some light on this problem.

\section{Acknowledgements}

We wish to thank P.P. Kulish for discussion. J.A. thanks LAPTH Annecy for their hospitality, and particularly the organizers of RAQIS 2005 for their remarkable achievement under such unhappy circumstances. Both authors fondly remember and sorely miss our friend and collaborator Daniel Arnaudon.

\section{References}

[1] I. Cherednik, "Factorizing particles on half-line and root systems", Theor. Math. Phys. 61 (1984) 977.

[2] E.K. Sklyanin, "Boundary conditions for integrable quantum systems", J. Phys. A21 (1988) 2375.

[3] P. P. Kulish, R. Sasaki, "Covariance Properties of Reflection Equation Algebras", Prog. Theor. Phys. 89 (1993), 741, hep-th/9212007

[4] J.M.Maillet; Phys. Lett. B 162 (1985), 137; L. Freidel, J.M. Maillet," On classical and quantum Integrable Field Theories associated to Kac Moody current algebras", Phys. Lett. B 263 (1992), 403

[5] L. Freidel and J.M. Maillet, "Quadratic algebras and integrable systems", Phys. Lett. B262 (1991) 278.

[6] J. Donin and A.I. Mudrov, "Reflection equation, twist and equivalent quantization", math-qa/0204295 J. Donin, P.P. Kulish and A.I. Mudrov, "On universal solutions to reflection equation", Lett. Math. Phys. 63 (2003) 179, math-qa/0210242.

[7] R. Malara, A. Lima-Santos, "On $A_{n-1}^{(1)}, B_{n}^{(1)}, C_{n}^{(1)}, D_{n}^{(1)}, A_{2 n}^{(2)}, A_{2 n-1}^{(2)}$ and $D_{n+1}^{(2)}$ Reflection K-Matrices", nlin.SI/0412058. 
[8] A. Kundu, "Exact Bethe ansatz solution of non ultralocal quantum mKdV model", Mod. Phys. Lett. A10 (1995), 2955; L. Hlavaty, "Quantum Braided groups", J. Math. Phys. 35 (1994), 2560.

[9] D. Arnaudon, J. Avan, N. Crampé, A. Doikou, L. Frappat and E. Ragoucy, "Classification of reflection matrices related to (super) Yangians and appplications to open spin chains", math-qa/0304150, to appear in Nucl. Phys. B.

[10] L. Mezincescu and R.I. Nepomechie, "Fusion procedure for open chains", J. Phys. A25 (1992) 2533; L. Mezincescu, R.I. Nepomechie and V. Rittenberg, "Bethe Ansatz solution of the Fateev-Zamolodchikov quantum spin chain with boundary terms", Phys. Lett. A147 (1990) 70.

[11] G. Delius, N. McKay, "Quantum group symmetry in sine-Gordon and affine Toda field theories on the half-line", Commun.Math.Phys. 233 (2003) 173-190, hep-th/0112023; A. Doikou, "From affine Hecke algebras to boundary symmetries", Nucl.Phys. B725 (2005) 493-530, math-ph/0409060

[12] Ping Xu, "Quantum dynamical Yang-Baxter equation over a non-abelian base", Comm. Math. Phys. 226 (2002) 475.

[13] J. Donin, A.I. Mudrov, "Quantum groupoids and dynamical categories", math.QA/0311316, "Dynamical Yang-Baxter equation and quantum vector bundles", QA/0306028.

[14] P. Etingof, A. Varchenko, "Solutions of the quantum dynamical Yang-Baxter equation and dynamical quantum groups", Comm. Math. Phys 196 (1998), 591, q-alg/9708015 B. Enriquez, P. Etingof, "Quantization of classical dynamical $r$-matrices with nonabelian base", math.QA/0311224: P. Etingof, "On the dynamical Yang-Baxter equation", Proceedings of the ICM, Beijing 2002, vol. 2, 555-570, math.QA/0207008

[15] J.L. Gervais and A. Neveu, "Novel triangle relations and absence of tachyons in Liouville string field theory", Nucl. Phys. B238 (1984) 125; G. Felder, "Conformal field theory and integrable systems associated to elliptic curves", hep-th/9407154.

[16] J. Avan, O. Babelon and E. Billey, "The Gervais-Neveu-Felder equation and quantum Calogero-Moser systems", Comm. Math. Phys. 178 (1996) 281.

[17] P.P. Kulish, "Yang-Baxter equation and reflection equations in integrable models", Lectures in Schladming School of Theoretical Physics (March 1995), hep-th/950\%0\%; Roger E. Behrend, Paul A. Pearce, David L. O'Brien, "Interaction-Round-a-Face Models with Fixed Boundary Conditions: The ABF Fusion Hierarchy", J. Stat. Phys. 84 (1996) 1, hep-th/9507118; H. Fan, B.-Y. Hou, K. J. Shi: Nucl. Phys. B 496 (1997), 551.

[18] R.J. Baxter, "Eight-Vertex model in Lattice Statistics and One-dimensional Anisotropic Heisenberg Chain; II: Equivalence to a Generalized Ice-type Model", Ann. Phys. 76 (1973), 25-47; "Exactly solved models in Statistical Mechanics", Academic Press, London, 1982.

[19] P.P. Kulish, A.I. Mudrov, "Dynamical reflection equation", math.QA/0405556.

[20] W.L. Yang, R. Sasaki, "Exact solution of $Z_{n}$ Belavin model with open boundary condition", Nucl.Phys. B679 (2004) 495-520, hep-th/0308127, Wen-Li Yang, Yao-Zhong Zhang, Mark D. Gould, "Exact solution of the XXZ Gaudin model with generic open boundaries", Nucl.Phys. B698 (2004) 503, hep-th/0411048.

[21] Z. Nagy, J. Avan, "Spin chains from dynamical quadratic algebras" ,J. Stat. Mech. 2 (2005) P03005, math.QA/0501029.

[22] Z. Nagy, J. Avan, G. Rollet, "Construction of dynamical quadratic algebras", 
Lett.Math.Phys. 67 (2004) 1-11, math.QA/0307026.

[23] G.E.Arutyunov, L.O.Chekhov, S.A.Frolov, "R-matrix Quantization of the Elliptic Ruijsenaars-Schneider model", q-alg/9612032.

[24] S.N.M. Ruijsenaars and H. Schneider, "A new class of integrable systems and its relation to solitons", Ann. Phys. 170 (1986) 370; S.N.M. Ruijsenaars, "Complete integrability of relativistic Calogero-Moser systems", Comm. Math. Phys. 110 (1987) 191.

[25] H. de Vega, A. Gonzalez Ruiz, "Boundary K-Matrices for the Six Vertex and the $n(2 n-$ 1) $A_{n-1}$ Vertex Models", J.Phys. A26 (1993) L519-L524, hep-th/9211114

[26] Z. Nagy, J. Avan, A. Doikou, G. Rollet, "Commuting quantum traces for quadratic algebras", J. Math. Phys. 46, 083516 (2005), math.QA/0403246.

[27] M. Mintchev, E. Ragoucy and P. Sorba; "Reflection-transmission algebras", hep-th/0303187.

[28] H. W. Braden, Takashi Suzuki, " $R$-matrices for the $n=2,3$ Elliptic Calogero-Moser Models", Lett.Math.Phys. 30 (1994) 147-158, hep-th/9312031.

[29] Omar Foda, K. Iohara, M. Jimbo, R. Kedem, T. Miwa, H. Yan, "An elliptic quantum algebra for $\widehat{s l}_{2}$ ", Lett.Math.Phys. 32 (1994) 259-268, hep-th/94030; M. Jimbo, H. Konno, S. Odake, J. Shiraishi, "Quasi-Hopf twistors for elliptic quantum groups", Transformation Groups 4 (1999), 303, q-alg/9712029 